\title{
Obstruction of Capillaries by circulating large Monocytes causes Multi Organ Dysfunction Syndrome (MODS) in Acute Pancreatitis
}

\section{Anika Wilden}

University Medicine Greifswald

Juliane Glaubitz

Department of Medicine A, University Medicine, University of Greifswald

Oliver Otto

University of Greifswald https://orcid.org/0000-0003-0280-5374

Doreen Biedenweg

Greifswald University Hospital

Matthias Nauck

Universitymedicine Greifswald

Matthias Mack

University Hospital Regensburg https://orcid.org/0000-0002-3157-7262

\section{Silvia Ribback}

Institute of Pathology, University of Greifswald, Greifswald

\section{Barbara Bröker}

University of Greifswald https://orcid.org/0000-0002-5020-8542

\section{Sabrina Freiin von Rheinbaben}

University Medicine

Markus Lerch

University Medicine

\section{Ali Aghdassi}

University Medicine

\section{Frank Weiss}

University Medicine Greifswald

Matthias Sendler ( $\square$ matthias.sendler@uni-greifswald.de)

University Medicine Greifswald

\section{Article}

Keywords: Monocytes, MODS, acute pancreatitis, CCR2 
Posted Date: April 27th, 2021

DOl: https://doi.org/10.21203/rs.3.rs-403798/v1

License: (c) (i) This work is licensed under a Creative Commons Attribution 4.0 International License. Read Full License 


\section{Abstract}

Acute pancreatitis (AP) is an inflammatory disorder, the severe form of which is burdened with high mortality. The pathogenesis of severity-driving organ manifestations, such as respiratory and renal failure, is unknown. We used samples from 300 pancreatitis patients and an experimental model of severe acute pancreatitis (SAP) to characterize severity-dependent cytokine profiles and resident/circulating immune cell populations by flow-cytometry and real-time- fluorescence and deformability-cytometry analysis. On functional, immunolabelling and in-vivo antibody-depletion experiments we confirmed the role of inflammatory cells in pancreatitis but found neither T-cells, Ly6g+neutrophils or granulocytic-myeloid-derived-suppressor-cells (gMDSC) but a massive mobilisation of CCR2+/CD11 b+-monocytes to be responsible for lung and kidney injury during SAP. Real-timefluorescence and deformability-cytometry analyses suggest, that the physical properties of monocytes, especially their large size, results in an obstruction of the fine capillary-systems of the lung or of the kidney glomeruli. Their selective depletion can represent a promising treatment strategy for pancreatitis as well as other inflammation-related disorders.

\section{Introduction}

Acute pancreatitis (AP) is one of the most common non-malignant gastroenterological disorders leading to hospitalisation of patients ${ }^{1}$. In most cases the disease has a mild self-limiting course, but $20 \%$ of patients develop severe acute pancreatitis, which is associated with systemic complications. Infected necrosis and/or persistent organ failure are the most perilous complications leading to significant morbidity and mortality ${ }^{1,2}$. AP can rapidly progress towards moderately severe or severe AP (SAP), significantly increasing the length of in-hospital stays and ultimately leading to significant healthcare expenditures ${ }^{3}$. Until today there is no established treatments for AP or SAP.

According to the revised Atlanta classification, the severe form of acute pancreatitis is defined by the occurrence of persistent organ failure or the presence of a systemic inflammatory response syndrome (SIRS) for longer than $48 \mathrm{~h}^{4}$. Pancreatitis-induced SIRS is the suggested trigger mechanism for organ damage, affecting mainly the pulmonary, the renal or the cardiovascular system ${ }^{2}$. While SIRS is held responsible for early organ damage like acute respiratory distress syndrome (ARDS) or acute kidney injury (AKI) ${ }^{2,5}$, the compensatory anti-inflammatory response syndrome (CARS) is believed to control infection of pancreatic necrosis and late organ failure. Schepers et al. recently showed a lack of correlation between mortality and the onset of early organ failure. This study also challenged the classical view of a biphasic or consecutive SIRS/CARS disease pattern. Beside the clinical observations also animal models of SAP suggest a parallel course of SIRS and CARS ${ }^{6}$, which could explain the observations by Schepers and co-workers ${ }^{7}$. A robust release of pro-inflammatory cytokines and chemokines can induce severe systemic inflammation, leading to multi-organ dysfunction syndrome $(\mathrm{MODS})^{5}$. In parallel, the adaptive immune system counteracts the developing cytokine storm by activating a suppressive immune response ${ }^{6,8,9}$, as has been shown for sepsis ${ }^{10,11}$. Pancreatitis-related 
organ complications frequently include the respiratory system ${ }^{2,7}$. IL-6 trans-signalling ${ }^{10}$ or MCP-1mediated monocyte/granulocyte mobilisation ${ }^{11}$ are associated with lung damage during AP. However, the precise pathomechanism behind pancreatitis-induced ARDS is not well understood, one reason being the lack of reproducible SAP animal models

Our aim for the present study was to investigate the cause of secondary organ damage/MODS in a mouse model of SAP induced by partial pancreatic duct ligation and hyperstimulation. In this model the mice developed the full spectrum of disease characteristics of SAP in humans ${ }^{12,13}$, including pancreatic necrosis and prominent damage of the respiratory and renal systems. Also, a parallel course of SIRS and CARS have been observed ${ }^{6}$. We used specific antibody treatment to analyse whether the depletion of neutrophils or monocytes affected the local and systemic immune responses, and how this might impact on the development of organ complications. Our results indicate a general disease mechanism via microcapillary occlusion from enlarged monocytes for the damage of secondary organs during severe inflammatory reactions, such as pancreatitis or sepsis, which offers a therapeutic option for the treatment of MODS.

\section{Results}

\section{Disease severity correlates with serum monocyte attracting chemokines}

304 pancreatitis patients that had been admitted to our university hospital between 2007 and 2020, were retrospectively classified according to the revised Atlanta criteria into mild, moderate, and severe pancreatitis. SAP patients frequently developed AKI and respiratory failure but also cardiovascular complications were observed (Fig. 1a). At the day of hospital admission leukocyte counts (Gpt/L) as well as $\mathrm{C}$-reactive protein (CRP) were significantly increased in these patients. In addition, serum creatinine, indicating kidney dysfunction, and serum lactate, indicating organ hypoperfusion as a sign of critical illness, were only significantly increased in the group of patients who developed SAP (Fig. 1b). Severe disease was also reflected by a significantly prolonged duration of hospitalisation (Fig. 1c). We next measured the concentration of serum chemokines using Cytometric bead arrays. Monocyte/macrophage attracting chemokines like MCP-1 (CCL-2), MIG (CXCL-9) or IP-10 (CXCL-10) were all significantly increased in SAP patients. Also neutrophil attracting chemokine IL-8 (CXCL-8) was elevated in a severity dependent manner (Fig. 1d). Anaphylatoxins, the cleavage products of complement proteins, are also known to have chemotactic characteristics, but we found no differences for $\mathrm{C} 3 \mathrm{a}, \mathrm{C} 4 \mathrm{a}$ and $\mathrm{C5a}$ with respect to disease severity (Fig. 1e). The correlation between selected chemokines and organ dysfunction was analysed by a correlation analysis of serum lactate and creatinine. Serum lactate showed a positive correlation with MCP-1, IL-8, MIG and IP-10 (Fig. 1f), whereas serum creatinine was significantly correlated with IP-10 and MIG (Fig. 1g). Blood leukocytes counts were positively correlated with serum lactate and creatinine levels, whereas CRP in serum showed only a weak correlation with creatinine but none of the chemokines (supplementary Fig. 1a-c). SAP is therefore associated with a significant mobilization of leukocytes into the vascular system, which may play an important role in the induction of secondary organ damage. 


\section{Organ damage in a mouse model of severe acute pancreatitis}

In the following experiments, we evaluated AP-associated organ damage in a severe pancreatitis animal model with high similarity to the human situation. Severe acute pancreatitis was induced by partial duct ligation with an additional hyperstimulation of caerulein ( $50 \mathrm{~g} / \mathrm{kg} / \mathrm{bodyweight}$ ) in C57BI/ 6 mice, as previously described ${ }^{6,12,13}$. H\&E staining of pancreas sections confirmed the development of a severe necrotizing pancreatitis $3 \mathrm{~d}$ after the ligation of the duct (Fig. 2a). This was paralleled by a significant increase in serum amylase and lipase activities, which are established markers of disease severity (Fig. 2b). Secondary organ damage was detected by a significant increase of myeloperoxidase activity in lung tissue, which is a consequence of infiltrating leucocytes (Fig. 2c). Histologic analysis of lung sections also showed a pronounced infiltration of leucocytes $3 \mathrm{~d}$ after the onset of pancreatitis, associated with increased alveolar wall thickness. Beside the infiltration of immune cells, oedema formation (black arrow) is a clear sign of acute lung injury (Fig. 2d). A histological examination of the lung volume by quantification of tissue area against alveolar space, showed a 20\% reduction $3 \mathrm{~d}$ after induction of AP (Fig. 2e). Furthermore, H\&E staining of kidney sections showed tubular dilatation (marked by rhomb) and intratubular deposits (marked by arrows), which are both histological signs of AKI and were present in tissue of mice $3 \mathrm{~d}$ after the onset of pancreatitis, but completely absent in healthy tissue (Fig. 2f $)^{14}$. Urinary accumulation of cystatin $\mathrm{C}$, a marker of $A K \mathrm{I}^{15}$, was detected by dot blot analysis (Fig. 2 g) in 3 out of 8 urine samples of pancreatitis animals, but not in control animals. Another indicator of kidney damage was the significantly increased proliferation of tubule cells shown by Ki67 labelling (Fig. 2h). Another indicator of AKI was the detection of a significantly increased expression of neutrophil gelatinase associated lipocalin (NGAL) ${ }^{16} 3 \mathrm{~d}$ after the onset of severe acute pancreatitis (Fig. $2 \mathrm{~h}$ ). In contrast to the rather mild pancreatitis model of hormonal (caerulein) hyperstimulation, the partial duct ligation in mice is associated with organ damage such as AKI and respiratory dysfunction, similar to the human situation.

\section{SAP-induced organ dysfunction is associated with infiltration of monocytes, but not neutrophils}

Hyperinflammation is believed to play a role in the induction of organ damage. We isolated leukocytes from lung tissue of SAP mice with respiratory dysfunction and evaluated infiltrating leukocytes by flow cytometry. CD4 + T-cells could only be detected in negligible quantities and we therefor analysed cells of the innate immune system, including mature neutrophils (Ly6g+/CD11b-), granulocytic myeloid derived suppressor cells gMDSCs (Ly6g+/CD11b+) and monocytes/macrophages (Ly6g-/CD11b+) by flow cytometry (Fig. 3a). While the number of neutrophils decreased $3 d$ after onset of pancreatitis, gMDSCs and monocytes/macrophages were significantly increased in lung tissue (Fig. 3b). Immunofluorescent labelling confirmed the presence of high numbers of CD11b + cells in lung tissue whereas only few Ly $6 \mathrm{~g}$ + cells could be detected (Fig. 3c). We also labelled CD11b + cells in tissue sections of lung and kidney, which confirmed that macrophages/monocytes are the major cell population infiltrating these organs. At $3 \mathrm{~d}$ after the onset of pancreatitis, CD11 + cells are visible within the alveolar septs of lung and the glomeruli of the kidney (Fig. 3d and 3e). 


\section{Leukocyte depletion ameliorates pancreatitis in mice}

In further experiments we analysed the specific role of leukocytes in the development of SAP. Therefore we depleted either neutrophils with an anti Ly $6 \mathrm{~g}$ antibody ${ }^{17}$ or monocytes via anti CCR2 antibody ${ }^{18} 1 \mathrm{~d}$ before and $1 \mathrm{~d}$ after onset of pancreatitis. Control mice were left untreated or received rat IgG2 Isotype antibody. The efficacy of the antibody-mediated depletion was confirmed by flow cytometry analysis of splenocytes (Fig. 4a). Treatment with anti-Ly $6 \mathrm{~g}$ antibody resulted in a total loss of Ly $6 \mathrm{~g}+$ neutrophils as well as of Ly $6 \mathrm{~g}+/ \mathrm{CD} 11 \mathrm{~b}+\mathrm{gMDSCs}$, whereas the treatment with anti-CCR2 antibody showed a significant reduction of CD11b + cells, but did not affect the population of Ly6g+/CD11b + gMDSCs (Fig. 4b-d). The effect of anti-Ly $6 \mathrm{~g}$ and anti-CCR2 treatment on pancreatic damage was investigated by histological evaluation. H\&E staining confirmed that the induction of pancreatic tissue damage was comparable in all animals, independent off leukocyte depletion (Fig. 4e). Labelling of neutrophils by anti-Ly6g antibodies revealed invasion of only few neutrophils in controls, but a complete absence of neutrophilic granulocytes after depletion with anti-Ly6g antibody in pancreatic tissue (Fig. 4e). Surprisingly, the depletion of monocytes by anti-CCR2 antibody did not influence the large number of CD68+ macrophages in the damaged pancreas (Fig. 4e). Quantitative analysis of neutrophil/macrophage counts confirmed a significant impact of the anti-Ly $6 \mathrm{~g}$ treatment but not of the anti-CCR2 treatment (Fig. $4 \mathrm{f}$ ). Serum amylase showed a significant reduction under both treatment conditions compared to the isotype antibody control group, whereas serum lipase was reduced in anti-Ly6g treated animals only (Fig. $4 \mathrm{~g}$ ).

\section{Macrophage-dependent pro-inflammatory response}

The systemic depletion of monocytes was not associated with a fast reduction in pancreatic macrophages, therefore pancreatic damage appeared less affected. In fact, only a small number of pancreatic macrophageswas positive for CCR2 and thereby sensitive to anti-CCR2 treatment. CCR2negative monocytes apparently started to proliferate (supplementary Fig. $2 a$ and $2 \mathbf{b}$ ). Macrophages orchestrate the activation of the adaptive immune system ${ }^{6,12}$, so it was not surprising that T-cell activation, as measured by the expression of CD25, CD69 or CD25+/Foxp3 $+T_{\text {reg }}$ cells, was not affected under treatment with anti-Ly6g or anti-CCR2 antibodies. Still, CD8a + cytotoxic T-cells were reduced after monocyte depletion (supplementary Fig. 2c). Pancreatic macrophages, but not infiltrating neutrophils, control the T-cell response via their cytokine profile $e^{6,12}$. The fact that tissue resident macrophages and not monocyte derived macrophages made up the majority of macrophages explains the missing effect of CCR2 depletion on T cell activation. Analysis of serum cytokines gave additional evidence that CCR2 depletion did not affect the systemic pro-inflammatory response (supplementary Fig. 3).

\section{Depletion of Monocytes ameliorates pancreatitis-induced organ damage}

In a next step we investigated the influence of neutrophil and monocyte depletion on pancreatitis-induced organ damage. We analysed the composition of leukocytes that had been isolated from lung tissue of mice by flow cytometry. Independent of the treatment with depleting antibodies we observed a dramatic reduction of Ly6g+/CD11b- mature neutrophils 3d after induction of pancreatitis (Fig. 5a). Neutrophil depletion by anti-Ly6g antibody resulted in no further reduction of Ly $6 \mathrm{~g}+/ \mathrm{CD} 11 \mathrm{~b}$ - neutrophils, but in a significant reduction of Ly6+/CD11b + gMDSCs. The depletion via anti-CCR2 antibody significantly 
decreased CD11b+/Ly6g- monocytes whereas the population of Ly6g+/CD11b + gMDSCs was not affected by the treatment (Fig. 5a and 5b). H\&E staining of lung tissue showed no significant differences between the isotype control group and both treatment groups (Fig. 5c). However, labelling of CD11b revealed a dramatic increase of CD11b + cells (gMDSC/monocytes) in isotype and anti-Ly6g treated mice but only a minor increase in mice which received the anit-CCR2 antibody (Fig. 5c and 5d).

Myeloperoxidase is expressed in large amounts in monocytes and neutrophils ${ }^{19}$ and as such a reliable marker of their tissue invasion. Measurements in lung tissue homogenate confirmed pancreatitis-induced elevated myeloperoxidase activities, which were significantly reduced in both antibody treatment groups compared to the isotype controls (Fig. 5e), To evaluate the respiratory function/dysfunction of these mice we analysed arterial blood samples and found in all animals suffering from SAP a significant reduction in oxygen saturation and increased serum lactate levels (Fig. 5f-g). Pancreatitis animals in general had decreased $\mathrm{pO}_{2}$ and glucose levels, whereas $\mathrm{pCO}_{2}$ and $\mathrm{ctHb}$ were elevated (supplementary Fig. 4a). Serum electrolytes remained unchanged (supplementary Fig. 4b). While the depletion of neutrophils had no beneficial effect on respiratory function, the monocyte depletion significantly increased oxygen saturation and reduced serum lactate levels (Fig. $5 f-g$ ). Serum creatinine levels were measured as indicator of AKI in mice. Mice which received isotype control or anti-Ly $6 \mathrm{~g}$ antibody showed a dramatic increase of serum creatinine levels, whereas creatinine levels from mice treated with anti-CCR2 antibody were comparable to untreated control mice (Fig. 5h). Immunohistochemical labelling of CD11b identified leukocytes in kidney glomeruli of control or anti-Ly6g treated, but not of anti-CCR2 treated animals (Fig. 5i).

\section{SAP mice have Increased numbers of enlarged blood monocytes}

Next, we analysed whether the identified CD11b + cells in histologic sections of lung and kidney were resident tissue cells, or whether these monocytes are circulating in the vascular system. Flow cytometric analysis of blood samples from SAP mice showed a significant increase of Ly $6 \mathrm{~g}+$ neutrophils and of CD11b + monocytes (Fig. 6a and b), which is in line with our observations in patients (Fig. 1). In contrast to the boosted response of the innate immune system, CD $4+$ T-helper cells were significantly reduced. (Fig. 6b). Monocytes and neutrophils, are both mobilized from bone marrow and associate with a more severe course of disease ${ }^{12,17,20}$. After induction of pancreatitis only a minority of circulating cells are Ly6g+/CD11b- mature neutrophils, whereas double positive Ly6g+/CD11b+ (gMDSCs) as well as Ly6g-/CD11b + monocytes were significantly increased, similar to our previous findings in spleen and lung tissue (Fig. 6c). As the monocyte depletion in our mouse model did not alter the local and systemic immune responses we speculated that a cell-mediated effect might be operative in the induction of organ damage. Beside their signalling involvement in immune regulation networks, the mechanical characteristics of myeloid derived cells have been discussed in a pathophysiological context in the recent literature ${ }^{21}$. In further experiments we isolated blood cells from mice and analysed their mechanical properties by real-time fluorescence and deformability cytometry (RT-FDC) after gating for 1 ) Ly6g+/CD11b- neutrophils, 2) Ly6g+/CD11b + gMDSCs and 3) Ly6g-/CD11b + monocytes (Fig. 6d) ${ }^{22}$. Deformation and cell size were analysed in Ly6g and CD11b labelled populations indicating a general increase in cell size (Fig. 6e). A comparison of six experimental replicates demonstrates a significant increase in cell size after $3 d$ of all investigated populations (Fig. 6f). In contrast, Ly $6 g$ + neutrophils as 
well as gMDSCs showed an increased deformation at 0d compared to CD11b+/Ly6g-monocytes (Fig. $6 \mathrm{~g}$ ). In fact, deformation of monocytes increased after onset of pancreatitis but did not reach the level of neutrophiles or gMDSCs. Applying an analytical model to our data enables to calculate the elastic modulus as an intrinsic material property ${ }^{23}$. The results indicate a significant increase for neutrophiles and gMDSCs $3 \mathrm{~d}$ after onset of pancreatitis, whereas the elasticity of monocytes was slightly, but not significantly smaller. During pancreatitis monocytes are mobilized in high numbers from the bone marrow and subsequently they circulate in the blood (Fig. 6b). The size of these monocytes is increased (Fig. 6f) but their flexibility is not increased accordingly (Fig. 6h). We hypothesized that these monocytes accumulate in the lung vascular system and obstruct the microcirculation, which results in respiratory dysfunction. In the next step, we therefore isolated monocytes from lung tissue and analysed them by RT$F D C^{22,24}$. After the induction of pancreatitis we observed a significant increase of cell size and deformation of monocytes in lung tissue, similar to our previous observation in whole blood (Fig. 7a-b). In to the same extent like monocytes isolated from blood, we found no significant differences in the elastic modulus (Fig. 7c). These findings associate pancreatitis with increasing monocyte size but maintained elastic modlus, leading to a congestion of monocytes in small vessels that causes organ damage. Immunofluorescent labelling of CD11b, Ly6g and VE-Cadherin supported this hypothesis. Increased numbers of $\mathrm{CD} 11 \mathrm{~b}+$ cells were observed within the vascular system of lung after onset of pancreatitis (Fig. 7d). CCR2 + monocytes accumulate within the tissue, whereas CD68 + resident alveolar macrophages are not increased (supplementary Fig. 5a). A similar picture we previously saw within the glomeruli of kidney in animals with SAP (supplementary Fig. 5b).

\section{Discussion}

The induction of a multi organ dysfunction syndrome during a sever course of acute pancreatitis ${ }^{4}$ is associated with dramatically increased morbidity and mortality and increasing health care utilization ${ }^{3}$. The underlying pathomechanisms behind pancreatitis associated MODS are unknown, and a causal therapy is missing. In our study we investigated organ damage in a pancreatitis mouse model which develops SIRS ${ }^{6,12}$ as well as MODS, both indicating severe acute pancreatitis according to the revised Atlanta classification ${ }^{4}$. Acute pancreatitis is associated with (mainly) necrosis of acinar cells, thus leading to local tissue damage and the initiation of an overwhelming pro-inflammatory immune response. Apparently, the extent of local damage has a critical influence on the strength of the inflammatory response and is therefore suggested to play an essential role in the induction of secondary organ damage and systemic complications ${ }^{2,5,10}$. It's the overwhelming immune response of acute pancreatitis which defines the severity of the disease course. An activation of the transcription factor NFKB in acinar cells occurs in parallel with intra acinar protease activation, and is an early event in disease manifestations ${ }^{25,26}$. The release of cytokines and chemokines then recruits immune cells to the pancreas where they contribute to the local damage $\mathrm{e}^{12,17,20,27,28}$ and enhance the immune response $\mathrm{e}^{6,29,30}$. Previous experiments suggest that SIRS and CARS in mouse models of SAP are not subsequent events, but occur in parallel ${ }^{6,8}$. Also, recent data from patients indicate a severity-dependent anti-inflammatory 
response in the presence of $\operatorname{SIRS}^{7,9}$. The activated immune response in the course of SAP is similar to other severe inflammatory responses such as sepsis ${ }^{31}$ or sever burn traumata ${ }^{32}$ which all may comprise the induction of MODS ${ }^{33,34}$. This suggests a common inflammatory response mechanism leading to secondary organ dysfunction. The most affected organs are the renal and the respiratory system which have one thing in common, their vascular system of micro-capillaries. A disturbed microcirculation therefore could contribute to organ failure ${ }^{33}$. We observed a significant increase of two cell populations of the innate immune system in the blood after the onset of pancreatitis: 1.) CD11b+/Ly $6 \mathrm{~g}$ - monocytes which could effectively be depleted by the usage of anti-CCR2 antibody ${ }^{18}$, and 2.) Ly6g+/CD11b + cells which represent gMDSCs ${ }^{35}$ and could successfully be depleted by anti-Ly $6 \mathrm{~g}$ antibody application. The pre-treatment with anti-Ly $6 \mathrm{~g}$ depleting antibody results in a complete loss of gMDSCs and Ly $6 \mathrm{~g}+$ neutrophils and attenuates the local pancreatic damage but has no effect on disease related organ dysfunction. It is well known that neutrophils contribute to acinar cell damage via the release of reactive oxygen ${ }^{20}$ species or pro-inflammatory cytokines like TNFa ${ }^{17}$. Our results confirm these findings but suggest that neutrophils are not involved in the initiation of systemic complications. Moreover, the number of Ly6g+/CD11b- mature neutrophils significantly decreased in lung tissue after the induction of pancreatitis, presumably as a consequence of an induced immunosuppression during SAP. Apparently, the removal of Ly6+/CD11b + gMDSCs and Ly6g+/CD11b- mature neutrophils in mice by depleting antibodies had no demonstrable effect on the systemic immune response because the number of mature neutrophils decreases during the induction of pancreatitis anyway.

In contrast, the depletion of monocytes by anti CCR2 antibody demonstrated that a macrophagemediated immune response is much more complex. CD11b+/Ly6g- monocytes were depleted from spleen and lung tissue of pancreatitis-induced mice, but the tissue-resident macrophages of the pancreas were not affected. Also, the systemic pro-inflammatory immune response did not differ between anti-CCR2 and isotype control mice, which supports the assumption that resident pancreatic macrophages initiate the development of SIRS/CARS by activating the adaptive immune system ${ }^{6}$. Tissue resident macrophages ${ }^{36,37}$ are independent of circulating monocytes. They start to proliferate in response to acinar cell damage and orchestrate the local and systemic immune response. While the depletion of CCR2 + monocytes did neither affect the systemic immune response, nor necrosis clearance by resident pancreatic macrophages, the development of organ dysfunction was significantly reduced. Monocytedepleted mice had better oxygen saturation and less elevated serum creatinine levels. Monocytes develop from precursor cells in the bone marrow and circulate in the blood ${ }^{38}$, whereas tissue resident

macrophages develop from invading monocytes starting with embryogenesis ${ }^{39}$. After transmigration into the tissue, they act as monocyte derived macrophages. In the adult, macrophages play a role in almost all inflammatory responses, including wound repair processes and it has been shown in adult guinea pigs that, when macrophage influx is blocked by administration of anti-macrophage serum, wound healing is severely impaired ${ }^{40}$. Following the induction of pancreatitis we saw a massive release of monocytes from bone marrow into the vascular system, but, surprisingly, no rapid transmigration into the site of pancreatic damage. The depletion of these circulating monocytes did not affect the local and systemic 
pro-inflammatory immune response, but significantly ameliorated organ complications. These findings suggest that the induction of MODS in severe pancreatitis is not mediated by an immunological signalling pathway but related to the physical properties of the monocytes ${ }^{21}$. Lung and kidney are characterised by microvascular systems and the passage of large cells, like monocytes, through these capillary systems requires a flexible adaptation to their small capillaries ${ }^{41}$. LPS activated monocytes acquire stiffer physical properties, leading to a prolonged retention in the capillary system of the lung ${ }^{42}$. Here we demonstrate that the induction of pancreatitis also changed the mechanical behaviour of myeloid derived cells dramatically. During pancreatitis, Ly6g + neutrophils, gMDSCs and CD11b + monocytes are all characterized by an increased cell size. However, neutrophils and gMDSCs revealed a high deformation compared to $\mathrm{CD} 11 \mathrm{~b}+$ monocytes. As a consequence, large numbers of these rather large and stiff monocytes remain circulating in the vascular system while they do not transmigrate into the pancreatic tissue. The depletion of these monocytes, but not of neutrophils and gMDSCs significantly ameliorated lung and kidney damage. A retrospective analysis of clinical data from patients demonstrated a clear correlation of leukocyte count and lactate, as marker for lung damage, or serum creatinine as marker for kidney damage. In addition, the serum level of the Monocyte attracting chemokines MCP-1, which is the ligand of CCR2, was significantly increased in patients with SAP compared to milder forms of the disease. This underlines the importance of monocytes for the disease severity and the induction of MODS in patients with SAP. Severe immune responses like in sepsis or severe trauma are also frequently associated with secondary organ damages like AKI or ARDS. We speculate that our observation is not a pancreatitis specific mechanism. Recent data suggest a parallel occurrence of SIRS and CARS during acute pancreatitis ${ }^{6}$, in a way that a severe pro-inflammation response, is counter-regulated by parts of the adaptive ${ }^{6,43}$ and innate immune system ${ }^{44}$ in order to prevent hyperinflammation and systemic shock. Monocytes are released from the bone marrow in response to the pancreatitis-induced pro-inflammation and enter the vascular system. A parallel compensatory systemic immunosuppressive reaction prevents their differentiation and, as a consequence, the cells remain in the vascular system. These large numbers of monocytes, isolated from blood as well as from lung tissue, which are characterized by increased size and unchanged elasticity are especially problematic for organs with small capillary networks like the lungs or the glomeruli of kidney. We propose that these monocytes represent a common pathomechanism and are the reason why severe immune reactions with a parallel SIRS/CARS lead to damaged renal and pulmonary system as it is known for pancreatitis, burn traumata and sepsis ${ }^{2,4,33,45}$. Recent genome wide association studies of lung tissue from Covid-19 patients identified an association of severe disease courses with increased CCR2 expression ${ }^{46}$. Monoclonal anti-CCR2 antibody therapy, which is already used for rheumatoid arthritis ${ }^{47}$ may be a treatment option against MODS during severe acute pancreatitis.

In summary, we could demonstrate a massive mobilisation of monocytes from the bone marrow in response to pro-inflammation during SAP. The number of circulating monocytes was dramatically increased after onset of pancreatitis and resulted in an impaired microcirculation in capillary networks. Beside the number of monocytes also their physical properties changed. Here, the cell size is increased without a compensatory increase in elasticity. This accumulation of monocytes in narrow capillaries of 
the lungs and glomeruli caused organ dysfunction, characterized by AKI and ARDS in a mouse model of SAP. The depletion of monocytes, but not of neutrophils or gMDSCs, was sufficient to significantly ameliorate lung and kidney damage. Monocytes represent a promising therapeutic target for the prevention of organ complications in SAP and possible other inflammation-related disorders affecting microcapillaries.

\section{Material And Methods}

\section{Animal Model}

Wildtype C57BI/6 mice were purchased from Charles River Laboratories (Sulzfeld, Germany). SAP was induced by partial duct ligation and an additional caerulein injection at day 2 (50 $\mathrm{mg} / \mathrm{kg}$ bodyweight), as described previously 6,8,12,13. To deplete Monocytes and Neutrophils mice were treated with anti-Ly6G antibody ${ }^{17}$ (BioXcell, Lebanon USA, BP0075-1), with rat anti-mouse-CCR2 antibody MC-2 $1^{18}$ (a kind gift from Matthias Mack, Regensburg Germany, lot 1480/02) and as control with isotype IgG2a (BioXcell, BP0089) or isotype IgG2b (BioXcell BE0090). Anti-Ly6G antibody and isotype IgG2a antibody were injected i.p. with $200 \mu \mathrm{g}$ in $200 \mu \mathrm{l}$ PBS one day before and the day after duct ligation. Anti-CCR2 antibody and Isotype IgG2b antibody were injected i.p. with $20 \mu \mathrm{g}$ in $200 \mu \mathrm{l} \mathrm{PBS}$ at the day of duct ligation and one and two days after surgery. Mice were sacrificed 72 hours after duct ligation and organs were used for analysis. Serum was stored at $-80^{\circ} \mathrm{C}$. Pancreas, lung and kidney were fixed in $4.5 \%$ formaldehyde and embedded in paraffin, embedded in TissueTec or frozen in liquid nitrogen for rtPCR analysis. Spleen and lung were taken for FACS analysis.

\section{Antibodies and Reagents}

The following antibodies were used for FACS staining, IHC staining and immunofluorescence staining: Anti-mouse CD4 BV650 (BioLegend 100546), anti-mouse-CD11b PerCP Cy55 (BioLegend, 101228), antimouse-Ly6G BV421 (BioLegend, 127628), anti-mouse-Ly6C BV605 (BioLegend 128036), anti-CD25-PECy7 (BioLegend, 102016), anti-CD69 BV510 (BioLegend, 104532), anti-CD8a BV605 (BioLegend, 100743), anti-FoxP3 APC (Miltenyi Biotec, 130-111-601), anti-mouse-CD11b (abcam, Ab133357), anti-mouse-CD68 (antibody-online, ABIN181836), anti-CCR2 (abcam, ab273050) anti-Ki67 (Bethyl, IHC-00375), anti-mouseCD11b (abcam, Ab133357), anti-mouse-Ly6g (abcam, Ab25377), anti-mouse-Cystatin C (Novus biologicals, NB100-1033), anti-VE-cadherin (abcam, ab7047-50) anti-rabbit-HRP (DAKO, K4003), antimouse-HRP (DAKO, K4001). Caerulein was obtained from Sigma Aldricht (C9026-1 MG, Munich, Germany), human myeloperoxidase from Calbiochem (Cat\# 475911).

\section{Flow cytometry analysis of spleen}

To analyse splenocytes, spleen was taken and mashed through a $70 \mu \mathrm{m}$ cell strainer. Cells were washed with PBS and centrifuged at $300 \mathrm{~g}$ for 6 minutes. To lyse erythrocytes pellet was resuspended in $1 \mathrm{ml}$ lysis buffer for 5 minutes. After washing with PBS and centrifugation at $300 \mathrm{~g}$ for 6 minutes cells were stained with fluorescence antibodies. Extracellular staining was performed with surface antibodies (1:50): AntiCD4, anti-CD11b, anti-Ly6G, anti-Ly6C, anti-CD25, anti-CD69, anti-CD8a. To block non-specific Fc mediated 
interactions cells were pre-treated with anti-mouse CD16/CD32 antibody for 5 min (1:50). Extracellular antibodies were incubated for 30 minutes at $4^{\circ} \mathrm{C}$. Cells were washed with PBS and centrifuged at $300 \mathrm{~g}$ for 6 minutes. Pellet was resuspended in 150 $\mu$ FACS buffer and measured immediately. Data were analysed with BD FACS DIVA Software and FlowJo.

\section{Flow cytometry analysis of lung}

To analyse leucocytes from lung, lungs were removed from mice and were dissociated according to the protocol of lung dissociation kit from Miltenyi (130-095-927, Bergisch Gladbach, germany). Staining for flow cytometry was performed as described in splenocyte staining. Data were analysed with BD FACS DIVA Software and FlowJo.

\section{Serum Amylase and Lipase}

Serum amylase and lipase measurement was performed with colorimetric assay from Roche/Hitacho (Amyl Ref 11876473316; Lip Ref 11821792216).

\section{Oxygen saturation of blood}

Arterial Blood was taken with a capillary from arteria carotis communis and was measured with BGA analysis machine (ABL90 FLEX, Radiometer $\mathrm{GmbH}$, Germany). Carotis communis was prepared as described previously. Parameters like $\mathrm{pO}_{2}, \mathrm{pCO}_{2}$, glucose, lactat, etc. were measured and analysed.

\section{MPO activity}

MPO measurement was performed like previously described ${ }^{6,17,48,49}$. Lung tissue was homogenized on ice in $20 \mathrm{mM}$ potassium phosphate buffer $(\mathrm{pH} \mathrm{7.4)}$ and centrifuged at $14.000 \mathrm{rcf}$. The pellet was resuspended in potassium phosphate buffer $(\mathrm{pH} 6.0)$ containing $0.5 \%$ cetyltrimethylammoniumbromide. Suspension was frozen and thawed in 4 cycles and centrifuged at $14.000 \mathrm{rcf}$. MPO activity was measured in $50 \mathrm{mM}$ potassium phosphate buffer ( $\mathrm{pH}$ 6.0) containing $0.53 \mathrm{mM}$ 0-dianisidine and 0.15 $\mathrm{mM} \mathrm{H}_{2} \mathrm{O}_{2}$. The MPO activity was measured as kinetic over time with a Spectramax Spectrophotometer (Molecular devices). Purified human myeloperoxidase was used as standard, the final MPO activity was calculated against protein content of the samples.

\section{Histology, Immunohistochemistry and Immunfluorescence staining}

For H\&E staining the lung, pancreas and kidney were embedded in paraffin, $3 \mu \mathrm{m}$ thick slides were cut by microtome. Slides were deparaffined in Xylol, and hydrated in methanol followed by decreased ethanol concentrations, hematoxylin and eosin staining was performed as described previously ${ }^{13,17}$. For lung analysis slides were scanned with slide scanner (Pannoramic MDI BF/FL; Sysmex, Norderstedt, Germany) and analysis were made with Software Pattern Quant from Sysmex Quant Center.

For immunohistochemistry staining slides were dewaxed and cooked in antigenretrieval solution (Dako target retrieval solution, ref. S1699). Rabbit anti-mouse-CD11b antibody (abcam, cambride UK, Ab133357) was used in a concentration of 1:400 over night at $4^{\circ} \mathrm{C}$. Anti-rabbit antibody was incubated for 1 hour at RT. DAB Kit (Vector, Burlingame USA, SK4100) was used to visualize CD11b positive cells. 
For immunofluorescence staining TissueTec embedded slides were used. Rabbit-anti-mouse-CD11b, Ly6g, CD68, CCR2 or VE-cadherin antibody was used as 1:400. Secondary antibody was used in a concentration of 1:200. DAPI was used for nucleus staining. All slides were scanned with Pannoramic slide scanner and analysis were made with Sysmex Quant Center Nucleus or Pattern.

\section{Cytokines in Serum}

The chemokines MCP-1, MIG, IP-10, IL8 and complement system anaphylatoxins C3a, C4a and C5a were measured in human serum from patients with severe, moderate and mild form of acute pancreatitis. Measurements were performed with CBA Kits from BD (Human Anaphylatoxin Kit 561418BD; Human Chemokine Kit 552990; BD San Jose, USA). Murine chemokines and cytokines were measured by BD cytometric bead array (CBA) mouse inflammation kit in serum of mice.

\section{Real Time Fluorescence and Deformability Cytometry}

Mechanical characterization of monocytes was performed in whole blood and lung monocytes isolated from pancreatic duct-ligated mice as well as untreated controls, respectively. For monocyte identification in whole blood, cells were labeled with Ly6g (APC conjugated) and CD11b (FITC conjugated) fluorescent antibodies. Lung monocytes were isolated (lung dissociation Kit, Miltenyi) by usage of monocytes isolation kit (EasySep mouse Monocyte Isolation Kit, Stemcell, 19861A). After isolation lung monocytes are resuspended in PBS (without $\mathrm{Ca}^{2+}$ and $\mathrm{Mg}^{2+}$ ) complemented with $0.6 \%(\mathrm{w} / \mathrm{v}$ ) Methylcellulose to a concentration of approximately $10 \times 10^{6}$ cells per $\mathrm{ml}$.

High-throughput mechanical analysis was done by real-time fluorescence and deformability cytometry using the AcCellerator system with fluorescence extension (Zellmechanik Dresden $\mathrm{GmbH}$ ) ${ }^{22,24}$. Briefly, the system consists of an inverted microscope, where a microfluidic chip with a $300 \mu \mathrm{m}$ long constriction is assembled on a $x y$-stage. We use a channel cross-section of $20 \mu \mathrm{m} \times 20 \mu \mathrm{m}$ for whole blood measurements and a $15 \mu \mathrm{m} \times 15 \mu \mathrm{m}$ cross-section for lung monocytes. In all measurements a flow rate of $0.06 \mu \mathrm{l} / \mathrm{s}$ is applied. For each condition data from several thousand monocytes has been obtained. Only cells are analysed with an area ratio $<1.05$ (ratio between actual cell area and cell area detected image analysis) to ensure a full representation of cell shape.

Derivation of elastic modulus was done using an analytical model published earlier ${ }^{23}$. Here, the flow profile around a moving cell is calculated assuming Stokes flow and deformation is predicted from coupling the resulting stress-distribution to the cell surface applying linear elasticity theory and assuming the cell being a homogeneous material.

Data were analysed with the Software ShapeOut.

\section{qrt-PCR}

RNA preparation was performed with Trizol as described before ${ }^{12}$. qrt-PCR was performed for NGal (5'CACCACGGACTACAACCAGTTCGC-3'; 5'-TCAGTTGTCAATGCATTGGTCGGTG-3'). As house-keeping gene 
5S (5'-GCCCGATCTCGTCTGATCTC-3'; 5'-GCCTATCAGCACCCGGTATTC-3') was analysed and markers were normalized to $5 \mathrm{~S}$.

\section{Statistical analysis}

The statistical evaluation as well as the graphical presentation of the experiments were carried out in GraphPad Prism and SigmaPlot. All graphs were generated with the standard error of the mean (SE). As significance test the two-sided students T-test for unpaired samples was used, all samples $p>0.05$ were classified as significant. Patients samples were analysed by Kruskal-Wallis test followed by Dunn's multiple comparison test, significant differences were defined by $p<0.05$, the correlation was tested by spearman rank order correlation. A statistical significant differences for more than 3 groups were tested by one way ANOVA followed by Tukey's multiple comparison test, or by Kruskal-Wallis test followed by Dunn's multiple comparison test. Statistical analysis of RT-FDC data has been performed using linear mixed models, where we compared two groups of experimental replicates, respectively ${ }^{50}$. While fixed effects account for the actual effect, i.e., increase or decrease in deformation, cell size or elastic modulus, random effects represent systematic or random measurement bias.

\section{Declarations}

\section{Acknowledgments:}

Specific author contributions: Concept of the study MS, FUW, AW. Data acquisition and interpretation: MS, AW, JG, AAA, DB, OO, MN, MM, SR, BMB, SFvB. Writing committee MS, FUW, AAA, MML. Correction of manuscript and approval of final version: all.

Funding: This work was supported by Deutsche Forschungsgemeinschaft (DFG SE 2702/2-1, AG 203/4-1 and GRK 1947), the PePPP center of excellence MV (ESF/14-BM-A55-0045/16) and the EnErGie/P2 Project (ESF/14-BM-A55-0008/18), the Bundesministerium für Bildung und Forschung (ZIK HIKE grant to OO under grant agreement 03Z22CN11) and the Deutsches Zentrum für Herz-Kreislauf-Forschung (Postdoc startup grant to 00 under grant agreement 81X3400107).

\section{Conflict-of-Interest}

$\mathrm{OO}$ is co-founder of Zellmechanik Dresden $\mathrm{GmbH}$ distributing the technology of real-time deformability cytometry.

\section{References}

1. van Dijk, S. M. et al. Acute pancreatitis: recent advances through randomised trials. Gut 66, 20242032 (2017).

2. Garg, P. K. \& Singh, V. P. Organ Failure Due to Systemic Injury in Acute Pancreatitis. Gastroenterology 156, 2008-2023 (2019). 
3. Peery, A. F. et al. Burden and Cost of Gastrointestinal, Liver, and Pancreatic Diseases in the United States: Update 2018. Gastroenterology 156, 254-272.e11 (2019).

4. Banks, P. A. et al. Classification of acute pancreatitis-2012: revision of the Atlanta classification and definitions by international consensus. Gut 62, 102-111 (2013).

5. Bhatia, M. \& Moochhala, S. Role of inflammatory mediators in the pathophysiology of acute respiratory distress syndrome. J. Pathol. 202, 145-156 (2004).

6. Sendler, M. et al. NLRP3 Inflammasome Regulates Development of Systemic Inflammatory Response and Compensatory Anti-Inflammatory Response Syndromes in Mice With Acute Pancreatitis. Gastroenterology 158, 253-269.e14 (2020).

7. Schepers, N. J. et al. Impact of characteristics of organ failure and infected necrosis on mortality in necrotising pancreatitis. Gut 68, 1044-1051 (2019).

8. Glaubitz, J. et al. Experimental pancreatitis is characterized by rapid T cell activation, Th2 differentiation that parallels disease severity, and improvement after CD4+ T cell depletion. Pancreatol. Off. J. Int. Assoc. Pancreatol. IAP Al (2020) doi:10.1016/j.pan.2020.10.044.

9. Zhang, R. et al. Expanded CD14hiCD16- Immunosuppressive Monocytes Predict Disease Severity in Patients with Acute Pancreatitis. J. Immunol. Baltim. Md 1950 202, 2578-2584 (2019).

10. Zhang, H. et al. IL-6 trans-signaling promotes pancreatitis-associated lung injury and lethality. J. Clin. Invest. 123, 1019-1031 (2013).

11. Frossard, J. L. et al. Role of CCL-2, CCR-2 and CCR-4 in cerulein-induced acute pancreatitis and pancreatitis-associated lung injury. J. Clin. Pathol. 64, 387-393 (2011).

12. Sendler, M. et al. Cathepsin B-Mediated Activation of Trypsinogen in Endocytosing Macrophages Increases Severity of Pancreatitis in Mice. Gastroenterology 154, 704-718.e10 (2018).

13. Sendler, M. et al. Complement Component 5 Mediates Development of Fibrosis, via Activation of Stellate Cells, in 2 Mouse Models of Chronic Pancreatitis. Gastroenterology 149, 765-776.e10 (2015).

14. Moeckel, G. W. Pathologic Perspectives on Acute Tubular Injury Assessment in the Kidney Biopsy. Semin. Nephrol. 38, 21-30 (2018).

15. Koyner, J. L. et al. Urinary cystatin $C$ as an early biomarker of acute kidney injury following adult cardiothoracic surgery. Kidney Int. 74, 1059-1069 (2008).

16. Nguyen, M. T. \& Devarajan, P. Biomarkers for the early detection of acute kidney injury. Pediatr. Nephrol. 23, 2151-2157 (2008).

17. Sendler, M. et al. Tumour necrosis factor a secretion induces protease activation and acinar cell necrosis in acute experimental pancreatitis in mice. Gut 62, 430-439 (2013).

18. Mack, M. et al. Expression and characterization of the chemokine receptors CCR2 and CCR5 in mice. J. Immunol. Baltim. Md 1950 166, 4697-4704 (2001).

19. Sugiyama, S. et al. Macrophage Myeloperoxidase Regulation by Granulocyte Macrophage ColonyStimulating Factor in Human Atherosclerosis and Implications in Acute Coronary Syndromes. Am. J. Pathol. 158, 879-891 (2001). 
20. Gukovskaya, A. S. et al. Neutrophils and NADPH oxidase mediate intrapancreatic trypsin activation in murine experimental acute pancreatitis. Gastroenterology 122, 974-984 (2002).

21. Bashant, K. R. et al. The mechanics of myeloid cells. Biol. Cell 112, 103-112 (2020).

22. Rosendahl, P. et al. Real-time fluorescence and deformability cytometry. Nat. Methods $15,355-358$ (2018).

23. Mietke, A. et al. Extracting Cell Stiffness from Real-Time Deformability Cytometry: Theory and Experiment. Biophys. J. 109, 2023-2036 (2015).

24. Otto, O. et al. Real-time deformability cytometry: on-the-fly cell mechanical phenotyping. Nat. Methods 12, 199-202, 4 p following 202 (2015).

25. Gukovsky, I., Gukovskaya, A. S., Blinman, T. A., Zaninovic, V. \& Pandol, S. J. Early NF-kappaB activation is associated with hormone-induced pancreatitis. Am. J. Physiol. 275, G1402-1414 (1998).

26. Steinle, A. U., Weidenbach, H., Wagner, M., Adler, G. \& Schmid, R. M. NF-kappaB/Rel activation in cerulein pancreatitis. Gastroenterology 116, 420-430 (1999).

27. Wu, J. et al. Macrophage phenotypic switch orchestrates the inflammation and repair/regeneration following acute pancreatitis injury. EBioMedicine 58, 102920 (2020).

28. John, D. S. et al. Deficiency of cathepsin C ameliorates severity of acute pancreatitis by reduction of neutrophil elastase activation and cleavage of E-cadherin. J. Biol. Chem. 294, 697-707 (2019).

29. Barreto, S. G. et al. Critical thresholds: key to unlocking the door to the prevention and specific treatments for acute pancreatitis. Gut 70, 194-203 (2021).

30. Mayerle, J. et al. Genetics, Cell Biology, and Pathophysiology of Pancreatitis. Gastroenterology 156, 1951-1968.e1 (2019).

31. Hotchkiss, R. S., Monneret, G. \& Payen, D. Sepsis-induced immunosuppression: from cellular dysfunctions to immunotherapy. Nat. Rev. Immunol. 13, 862-874 (2013).

32. Xiao, W. et al. A genomic storm in critically injured humans. J. Exp. Med. 208, 2581-2590 (2011).

33. Rossaint, J. \& Zarbock, A. Pathogenesis of Multiple Organ Failure in Sepsis. Crit. Rev. Immunol. 35, 277-291 (2015).

34. Wang, H. \& Ma, S. The cytokine storm and factors determining the sequence and severity of organ dysfunction in multiple organ dysfunction syndrome. Am. J. Emerg. Med. 26, 711-715 (2008).

35. Bronte, V. et al. Recommendations for myeloid-derived suppressor cell nomenclature and characterization standards. Nat. Commun. 7, 12150 (2016).

36. Zhu, Y. et al. Tissue-Resident Macrophages in Pancreatic Ductal Adenocarcinoma Originate from Embryonic Hematopoiesis and Promote Tumor Progression. Immunity 47, 323-338.e6 (2017).

37. Davies, L. C., Jenkins, S. J., Allen, J. E. \& Taylor, P. R. Tissue-resident macrophages. Nat. Immunol. 14, 986-995 (2013).

38. Jakubzick, C. V., Randolph, G. J. \& Henson, P. M. Monocyte differentiation and antigen-presenting functions. Nat. Rev. Immunol. 17, 349-362 (2017). 
39. Hopkinson-Woolley, J., Hughes, D., Gordon, S. \& Martin, P. Macrophage recruitment during limb development and wound healing in the embryonic and foetal mouse. J. Cell Sci. 107 (Pt 5), 11591167 (1994).

40. Leibovich, S. J. \& Ross, R. The role of the macrophage in wound repair. A study with hydrocortisone and antimacrophage serum. Am. J. Pathol. 78, 71-100 (1975).

41. Dupire, J., Puech, P.-H., Helfer, E. \& Viallat, A. Mechanical adaptation of monocytes in model lung capillary networks. Proc. Natl. Acad. Sci. U. S. A. 117, 14798-14804 (2020).

42. Doherty, D. E., Downey, G. P., Schwab, B., Elson, E. \& Worthen, G. S. Lipolysaccharide-induced monocyte retention in the lung. Role of monocyte stiffness, actin assembly, and CD18-dependent adherence. J. Immunol. Baltim. Md 1950 153, 241-255 (1994).

43. Sakaguchi, S. Naturally arising CD4+ regulatory t cells for immunologic self-tolerance and negative control of immune responses. Annu. Rev. Immunol. 22, 531-562 (2004).

44. Gabrilovich, D. I. \& Nagaraj, S. Myeloid-derived suppressor cells as regulators of the immune system. Nat. Rev. Immunol. 9, 162-174 (2009).

45. Sapan, H. B. et al. Pattern of cytokine (IL-6 and IL-10) level as inflammation and anti-inflammation mediator of multiple organ dysfunction syndrome (MODS) in polytrauma. Int. J. Burns Trauma 6, 37-43 (2016).

46. Pairo-Castineira, E. et al. Genetic mechanisms of critical illness in COVID-19. Nature 591, 92-98 (2021).

47. Vergunst, C. E. et al. Modulation of CCR2 in rheumatoid arthritis: a double-blind, randomized, placebo-controlled clinical trial. Arthritis Rheum. 58, 1931-1939 (2008).

48. Aghdassi, A. A. et al. Cathepsin D regulates cathepsin B activation and disease severity predominantly in inflammatory cells during experimental pancreatitis. J. Biol. Chem. 293, 10181029 (2018).

49. Sendler, M. et al. Cathepsin B Activity Initiates Apoptosis via Digestive Protease Activation in Pancreatic Acinar Cells and Experimental Pancreatitis. J. Biol. Chem. 291, 14717-14731 (2016).

50. Herbig, M., Mietke, A., Müller, P. \& Otto, O. Statistics for real-time deformability cytometry: Clustering, dimensionality reduction, and significance testing. Biomicrofluidics 12, 042214 (2018).

\section{Figures}




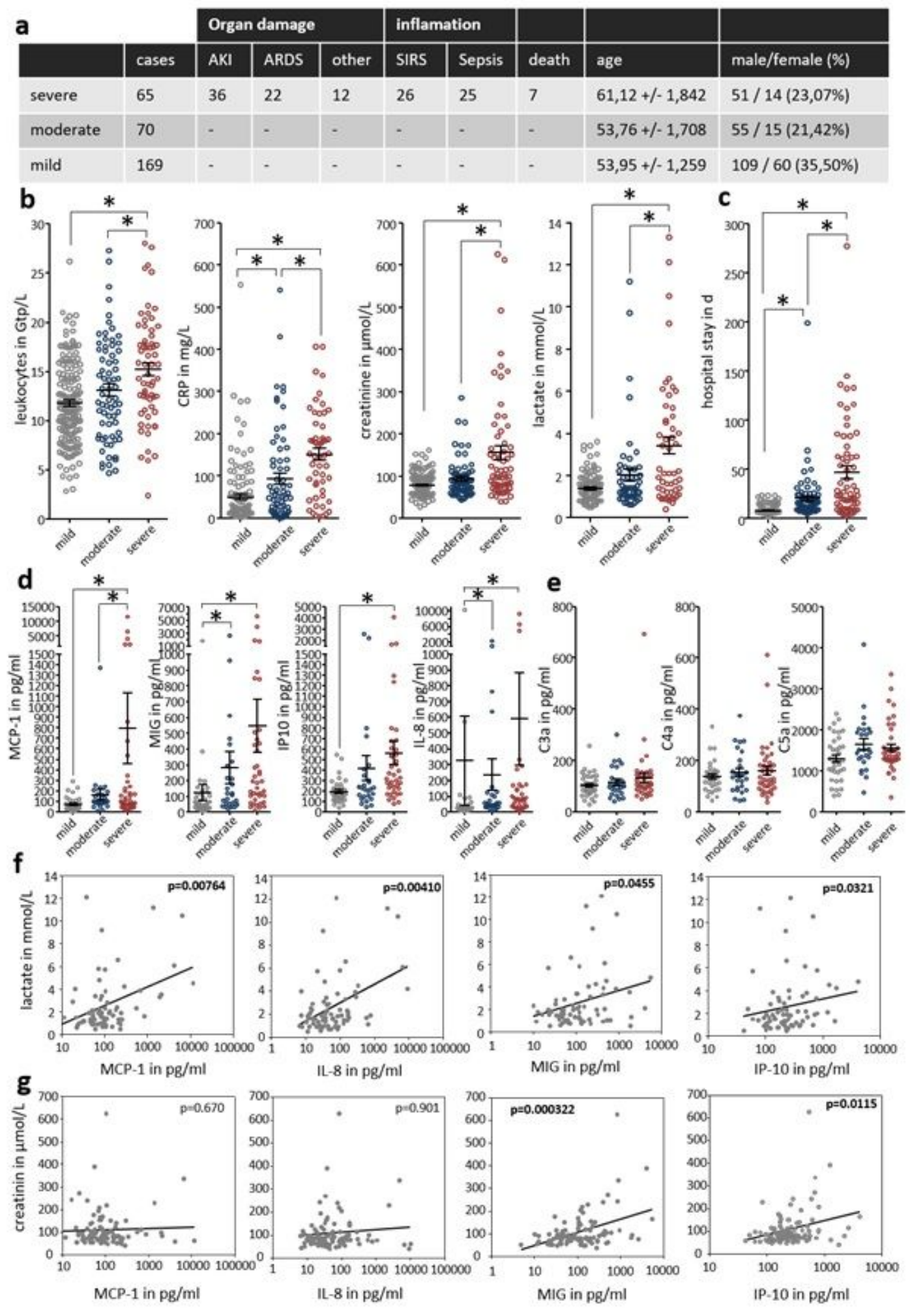

\section{Figure 1}

Patients were grouped based on the revised Atlanta classification (a). Persistent organ failure or SIRS/sepsis are characteristics of a severe course. Acute kidney injury (AKI 55.4\%) and acute lung injury (ALI 33.8\%) were the most frequent organ complications. Clinical parameters were evaluated from the day of admission (b). Inflammatory markers (leukocytes count and C-reactive protein (CRP)), serum lactate and creatinine levels were significantly increased in SAP patients. (b). Severe disease is also 
reflected by a significantly prolonged hospital stay (c). Serum chemokines and complement cleavage products were analysed in 104 AP patients (d-e). Chemokines, MCP-1, MIG, IP-10 and IL-8 were significantly increased in SAP (d), whereas complement factors $\mathrm{C} 3 \mathrm{a}, \mathrm{C} 4 \mathrm{a}$ and $\mathrm{C} 5 \mathrm{a}$ show no severity related increase (e). Patients samples were analysed by Kruskal-Wallis test followed by Dunn's multiple comparison test, significant differences were defined by $\mathrm{p}<0.05$ and marked by asterisk. Correlation analyses were performed for serum lactate $(\mathrm{f})$ and serum creatinine $(\mathrm{g})$ to test a possible relationship between serum chemokines and markers of organ dysfunction. A correlation was tested by spearman rank order correlation and $p$ values are shown in each graph. 


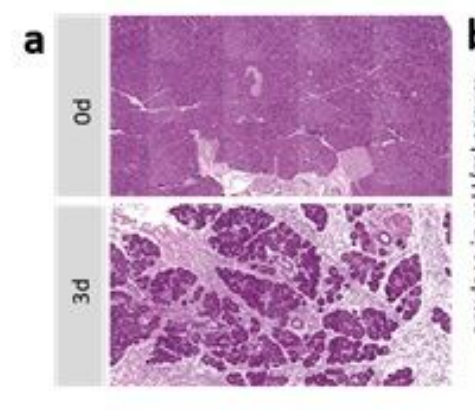

d

od
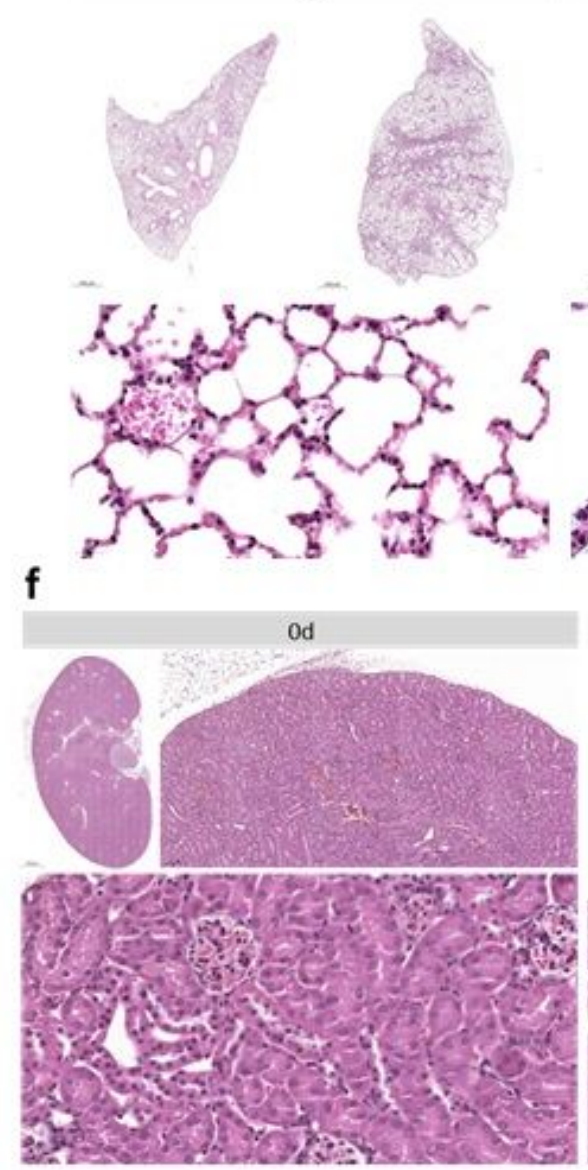

h

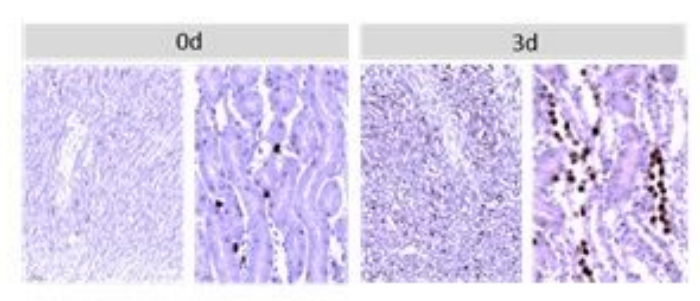

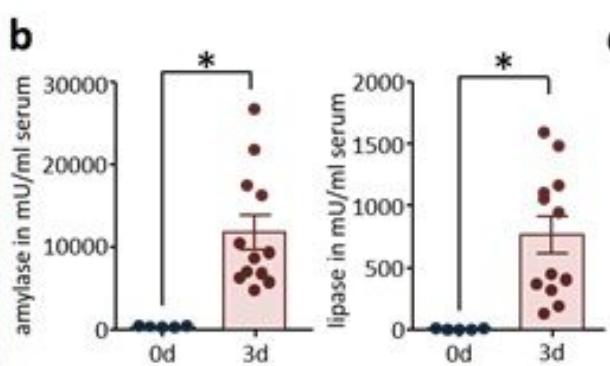
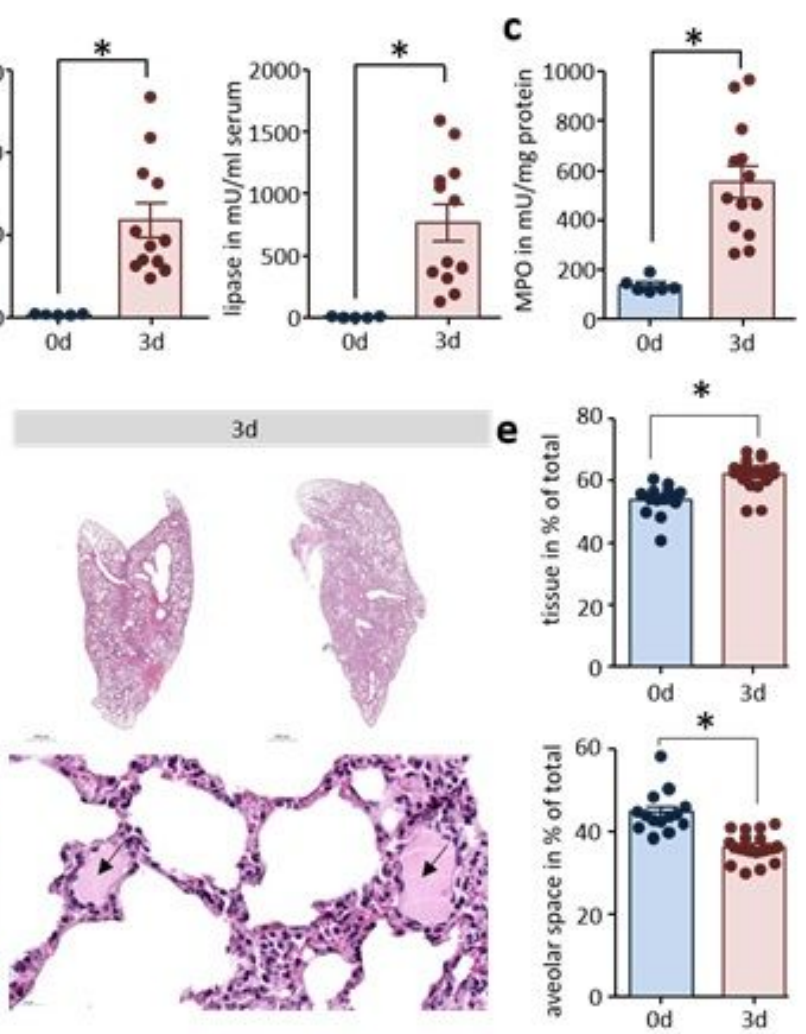

g
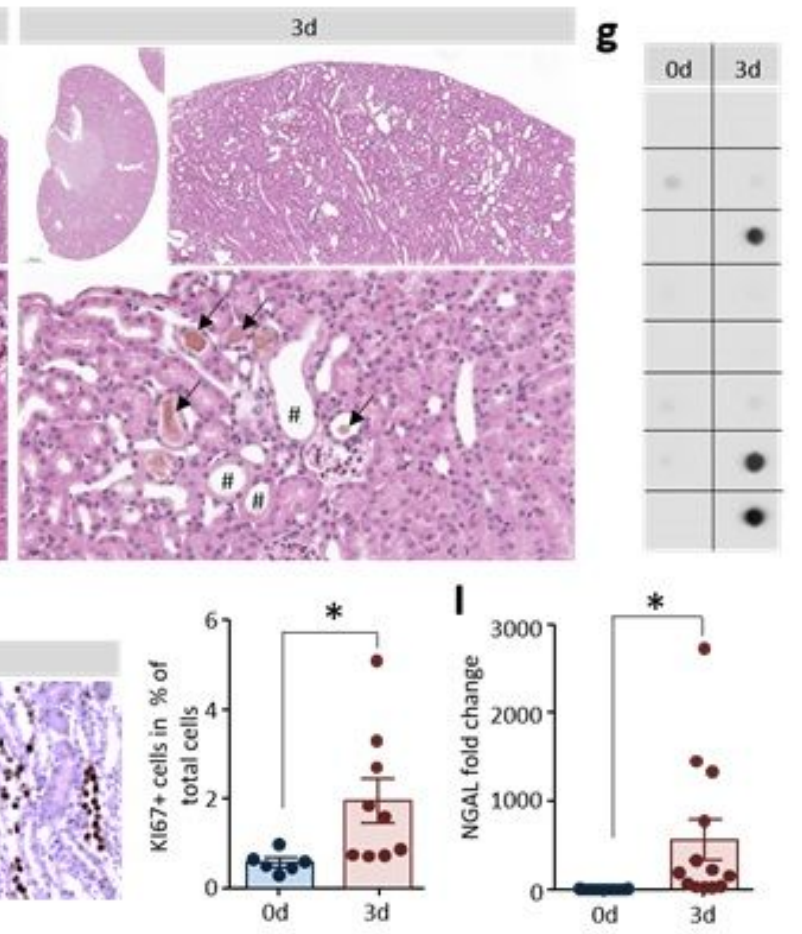

\section{Figure 2}

Organ damage was investigated in a mouse model of SAP. Pancreatitis was induced in C57BI/ 6 mice by partial duct ligation, animals were sacrificed $72 \mathrm{~h}$ later. H\&E staining of pancreatic sections (a) illustrate organ damage, inflammation and acinar cell necrosis. Serum amylase and lipase were significantly increased $3 \mathrm{~d}$ after onset of disease (b). Organ damage was investigated in lung and kidney of mice. Myeloperoxidase (MPO) activity a marker of the presence of inflammatory cells was elevated in lung 
tissue (c). H\&E staining showed a clear increase of wall thickness and oedema within the alveolus (black arrows) (d). An analysis of the alveolar space/tissue volume ratio showed a significant decrease of the alveolar lung volume. (e). H\&E staining of kidney sections showed also clear signs of early tissue damage $3 d$ after induction of pancreatitis: tubular dilatation (rhomb) and hyaline casts (black arrows) (f). Dot blot analysis of cystatin $C$ in urine samples of mice ( $n=8 / 8$ for controls and SAP) were positive in 3 out of 8 mice with SAP $(\mathrm{g})$. Tubular dilatation was associated with increased cell proliferation (shown by Ki67 positive nuclei). Quantification of Ki67 staining showed a significant increase of tubular cell proliferation (h). RT-PCR analysis of NGAL in kidney tissue showed a significant increase $3 \mathrm{~d}$ after onset of pancreatitis (i). All graphs represent 5 or more animals per group. Statistically significant differences were tested by unpaired students t-test for independent samples and significance levels of $p<0.05$ are marked by an asterisk.

a

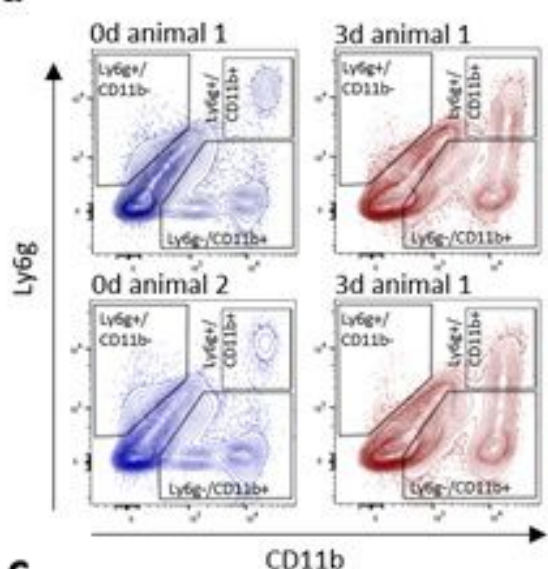

b

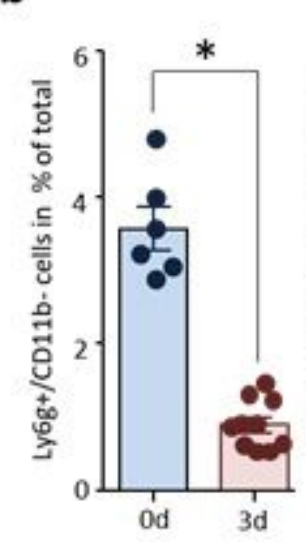

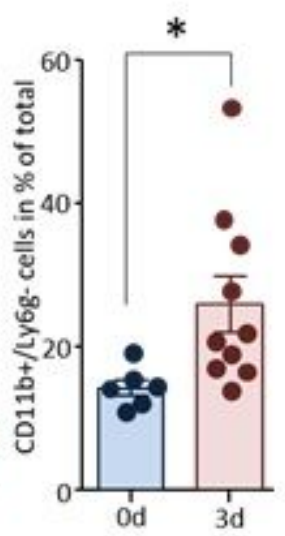

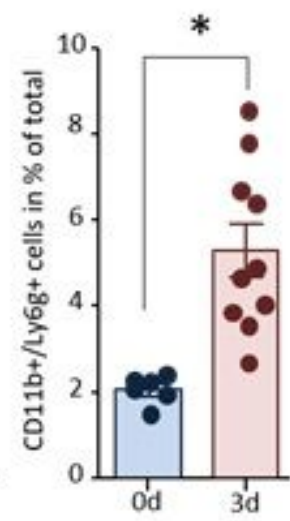

C
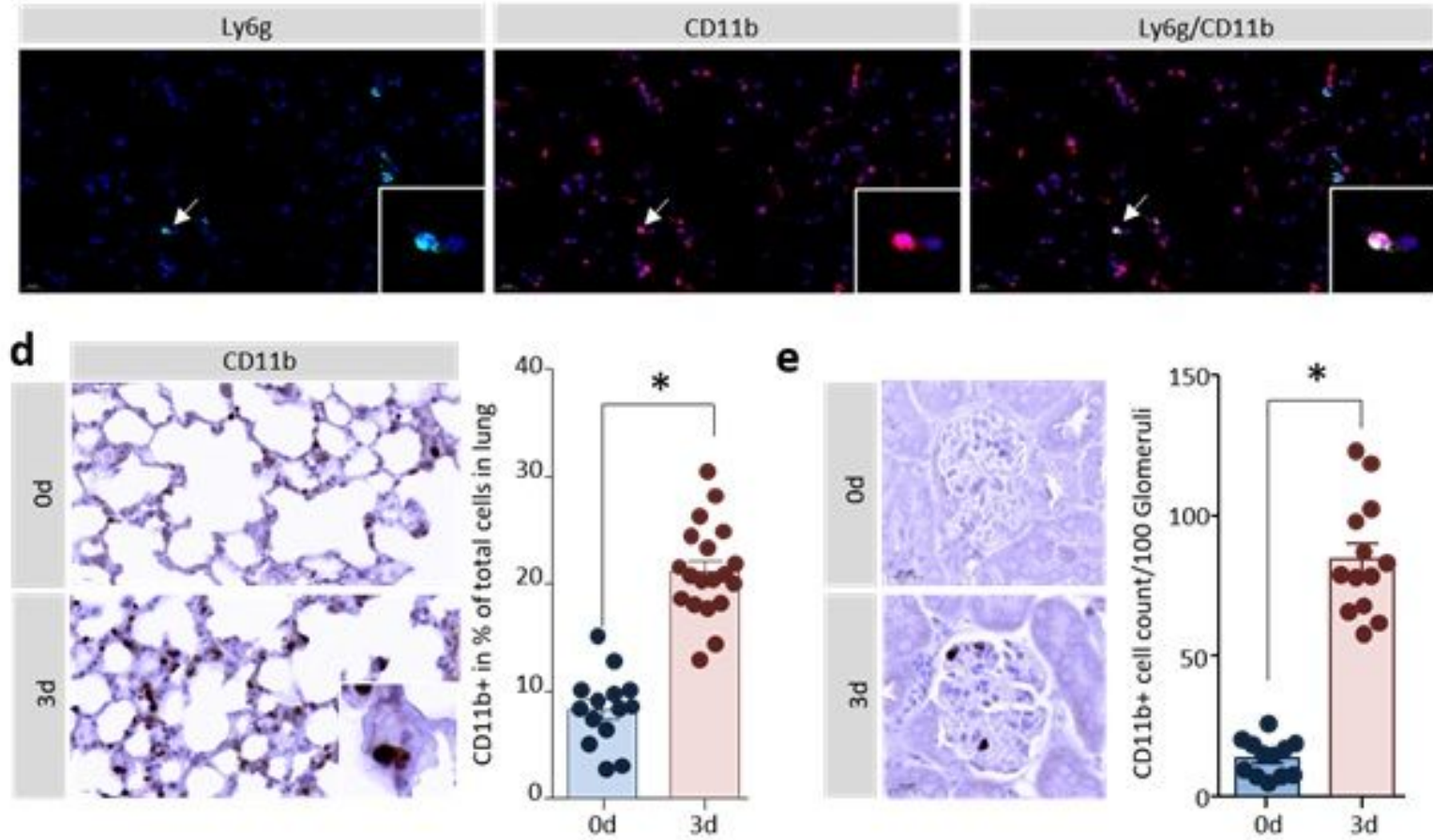

e
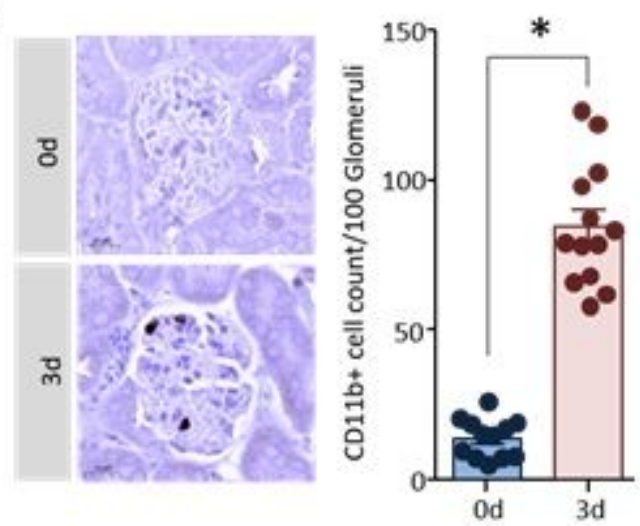

Figure 3 
Classification of leukocytes from lung tissue of mice by flow cytometry analysis of the surface markers Ly6g and CD11b (a). The number of Ly6g+/CD11b- mature neutrophils is decreased after the induction of pancreatitis, whereas the population of Ly6g+/CD11b+gMDSCs as well as Ly $6 \mathrm{~g}-/ C D 11 \mathrm{~b}+$ monocytes is significantly increased (b). This could also be shown by quantification of immunofluorescence labelling of Ly6g and CD11b in lung sections at 3d after onset of pancreatitis (c). CD11b labelling showed a clear redistribution of $C D 11 b+$ cells into the interalveolar septs. Cell counts of $C D 11 b+$ positive cells in lungs were significantly increased after the induction of pancreatitis in mice (d). Also, in kidney sections we observed a significant increase of CD11b+ cells in the glomerulus (e). All graphs represent 6 or more animals per group. Statistically significant differences were, tested by unpaired students t-test for independent samples and significance levels of $p<0.05$ are marked by an asterisk. 

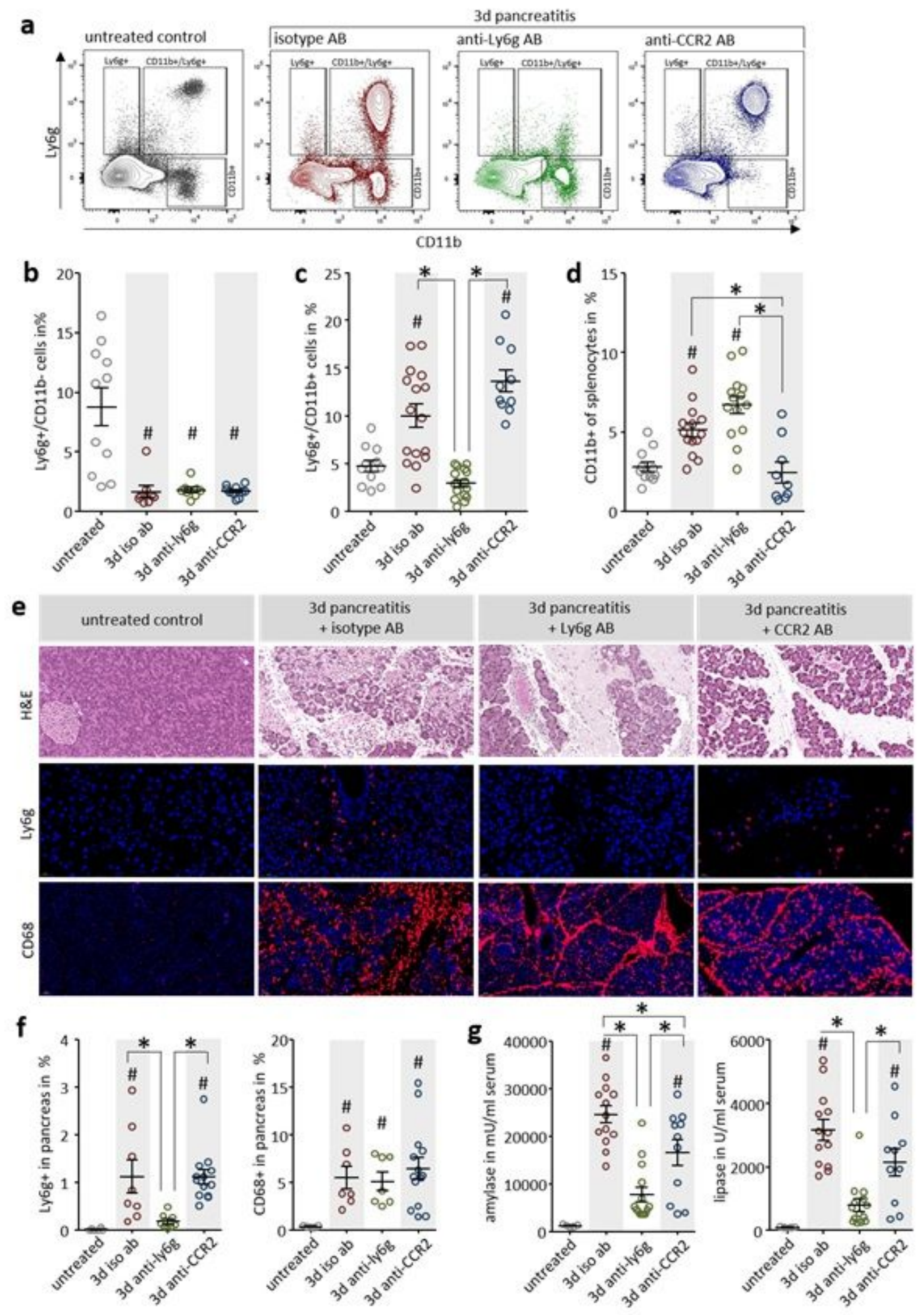

\section{Figure 4}

The SAP mouse model was utilized to analyse the effect of a depletion of neutrophils by anti-Ly $6 \mathrm{~g}$ antibody and the depletion of monocytes by anti-CCR2 antibody. Control groups were left untreated or received isotype antibody treatment. The efficiency of the depletion was proven by flow cytometry analysis of splenocytes with the surface markers Ly6g and CD11b to distinguish neutrophils (Ly6g+), gMDSCs (Ly6g+/CD11b+) and monocytes (CD11b+) (a). Mature Ly6g+/CD11b- neutrophils were 
significantly reduced in all animals after induction of pancreatitis, independent of the antibody treatment (b). An increase of Ly6g/CD11b double positive gMDSCs could be observed after onset of disease in isotype and anti-CCR2 treated mice, but not after anti-Ly6g antibody treatment (c). In contrast, the number of CD11b+/Ly6g-cells was increased during pancreatitis but not in the anti-CCR2 antibody treated group (d). Pancreas histology showed development of necrosis in all pancreatitis animals except in untreated controls. Immunofluorescent Ly6g-labelling showed the absence of $\mathrm{Ly} 6 \mathrm{~g}+$ neutrophils in pancreatic tissue of anti-Ly6g treated mice. Labelling by anti-CD68 showed a dramatic increase of macrophages in the pancreatic tissue of all mice with pancreatitis, even after the depletion of monocytes by anti-CCR2 treatment, (e). These observations were confirmed by quantitative evaluation of the immunofluorescencelabelled pancreatic sections ( $f$ ). Serum amylase and lipase activity reflected disease severity and showed a reduction a dramatic in the anti-Ly $6 \mathrm{~g}$ treated mice and a slight decrease in the anti-CCR2 treated animals $(\mathrm{g})$. All graphs represent 6 or more animals each group. A statistical significant differences was tested by one way ANOVA followed by Tukey's multiple comparison test, or by Kruskal-Wallis test followed by Dunn's multiple comparison test, A significance level of $p<0.05$ is marked by asterisk, rhombs indicate a significant difference to the untreated control. 

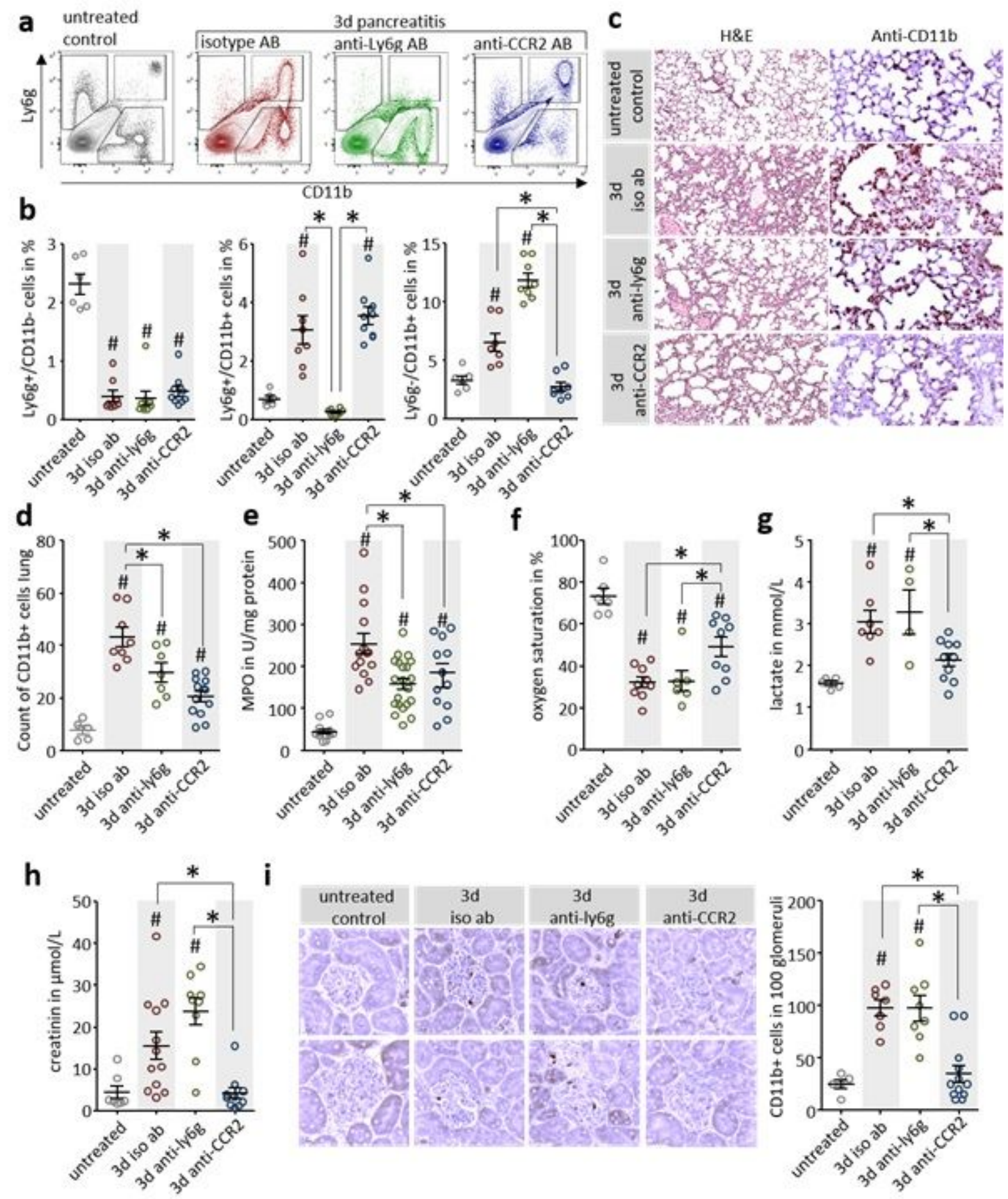

\section{Figure 5}

Anti-CCR2 treatment but not anti-Ly6g treatment ameliorates MODS during severe acute pancreatitis. Leukocytes were isolated from lung tissue and were analysed by flow cytometry using the surface markers Ly6g and CD11b (a). Three days after the onset of pancreatitis a significant reduction of Ly6g+/CD11b- mature neutrophils was observed in all treatment groups. In contrast, the population of Ly6g+/CD11b+gMDSCs was increased in lung tissue after the onset of pancreatitis, but the increase could be blocked by the anti-Ly6g treatment. Ly6g-/CD11b+ monocytes were also increased in 
pancreatitis mice and this increase was blocked by anti-CCR2 treated animals (b). H\&E staining of lung sections illustrates the pancreatitis-induced organ damage, and the specific labelling of CD11b cells confirmed the flow cytometry results (c) and showed significant differences after quantification (d). The pancreatitis induced increase of Myeloperoxidase activity in lung was attenuated in anti-Ly $6 \mathrm{~g}$ and antiCCR2 treated mice (e). Oxygen saturation in arterial blood samples of pancreatitis animals was reduced, but anti-CCR2 treatment improved the $\mathrm{s} 02$ ratio compared to iso-type or anti-Ly $6 \mathrm{~g}$ treated mice $(\mathrm{f})$. Increased serum lactate in pancreatitis reflects a shift to anaerobic metabolism which also is ameliorated in anti-CCR2 treated mice $(\mathrm{g})$. Elevated serum creatinine levels in pancreatitis animals indicate kidney dysfunction which could also be ameliorated by anti-CCR2 treatment $(h)$. Labelling of CD11b in kidney sections showed reduced numbers of CD11b+ cells located in the glomeruli following anti-CCR2treatment, and their quantitative analysis revealed significant differences in comparison to controls (i). All graphs represent 5 or more animals each group, significance was tested by one way ANOVA followed by Tukey's multiple comparison test, or by Kruskal-Wallis test followed by Dunn's multiple comparison test. A significance level of $p<0.05$ is marked by asterisk, rhombs indicate significant difference to the untreated control mice. 

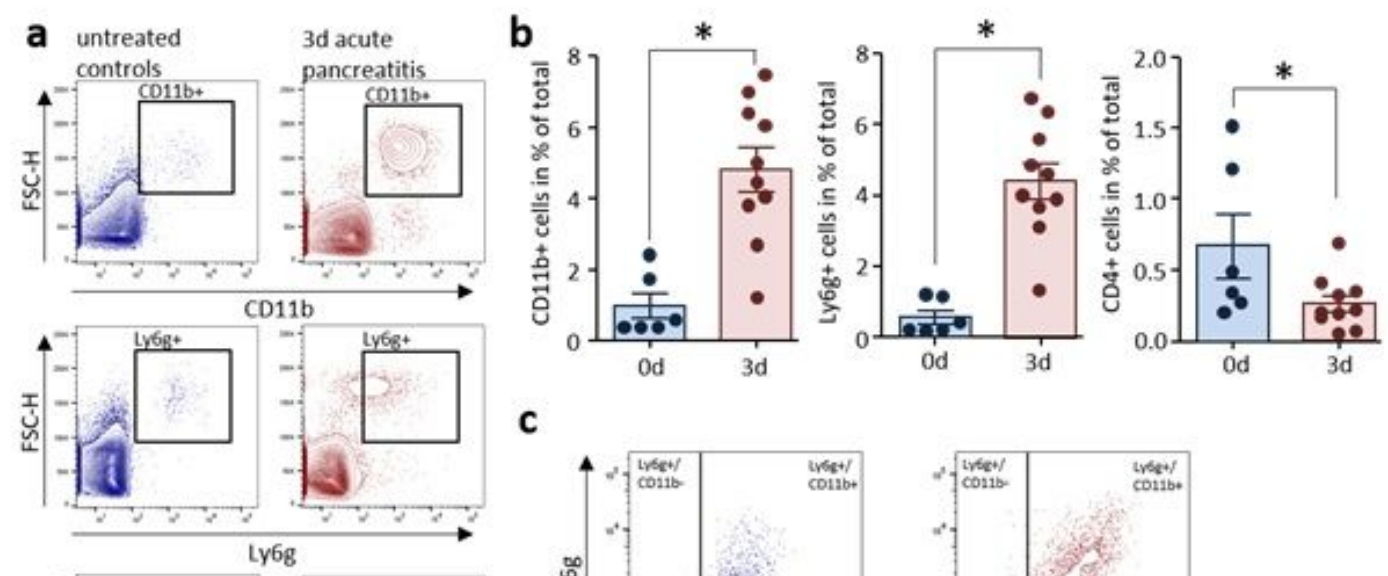

c
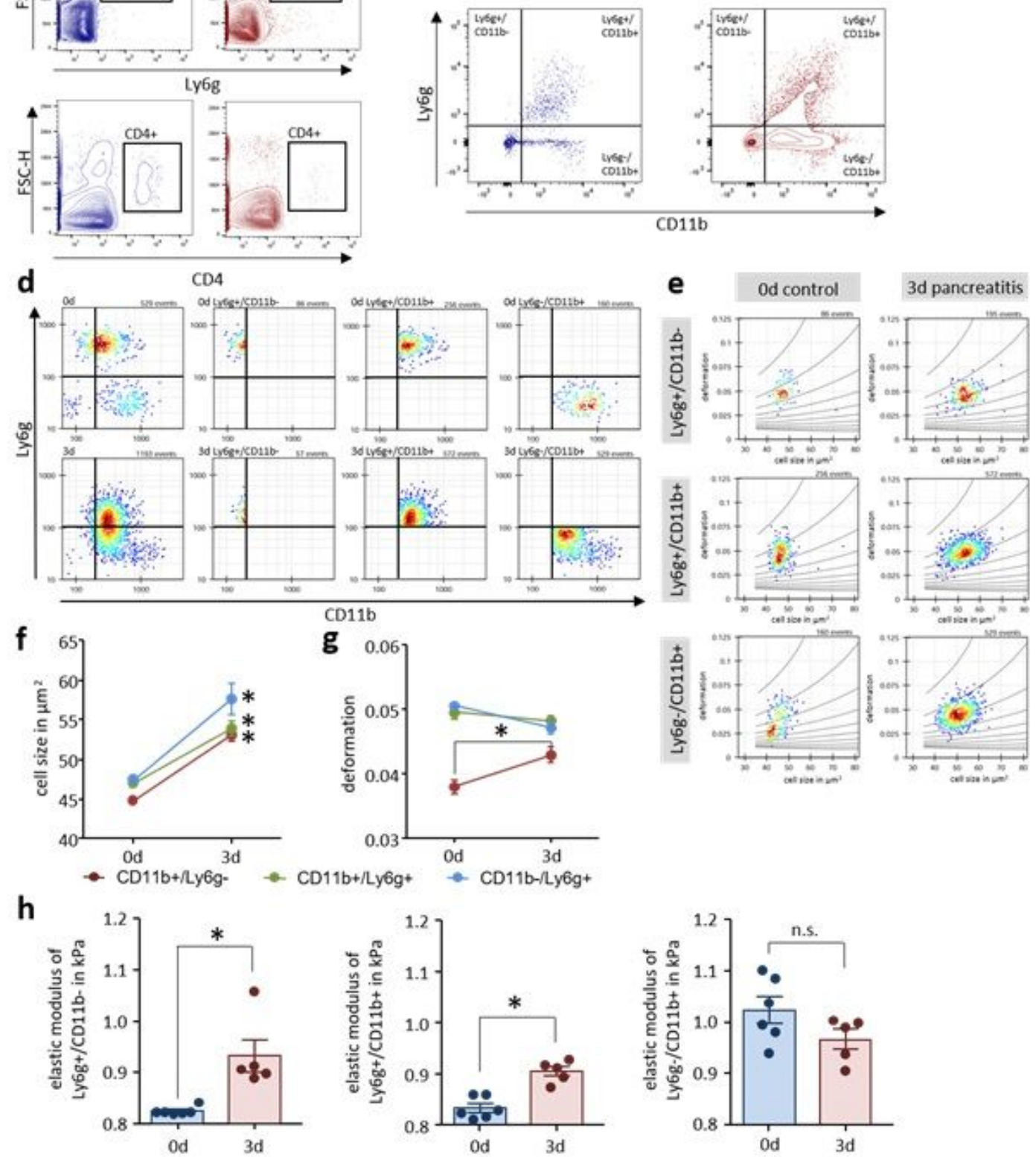

\section{Figure 6}

Leukocyte analysis from whole blood samples of SAP mice and controls. by Flow cytometry analysis showed a significant increase of Ly6g+ and CD11b+ cells whereas CD4+ T-lymphocytes were decreased during pancreatitis $(a, b)$. Ly6g+/CD11b+ MDSCs and Ly6g-/CD11b+ monocytes were significantly increased but not mature neutrophils, characterized by Ly6g+/CD11b- (c). The physical cell characteristics of these 3 populations was determined by real-time fluorescence and deformability 
cytometry (RT-FDC). Specific Ly6g and CD11b fluorescent antibody labelling was used to discriminate the 3 populations (neutrophils, gMDSCs and monocytes) (d). Scatter plots of deformation vs. cell size for Ly6g+/CD11b-, Ly6g+/CD11b+ and Ly6g-/CD11b+ monocytes using the gates shown in panel d (e). Comparing mean values of cells size and deformation indicates a size increase for all populations shown in (d) while an increase in deformation is only observed in CD11b+/Ly $6 \mathrm{~g}$ - monocytes, after induction of SAP, but not reaching the deformation of neutrophils and gMDSCs $(f, g)$. The elastic modulus increases significantly for neutrophils and gMDSCs but not for monocytes. All graphs represent 5 or more animals each group, significance was tested by unpaired, two tailed students t-test. RT-FDC data has been analysed using linear mixed models $(*, p<0.05)$. Figure 7 Monocytes isolated from lung tissue of pancreatitis 
a

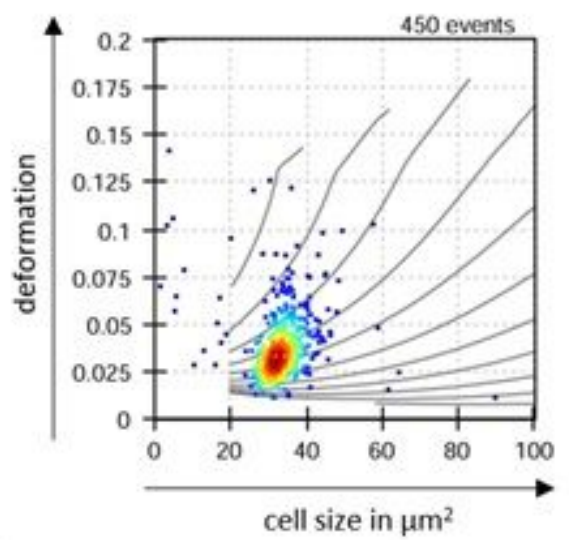

b
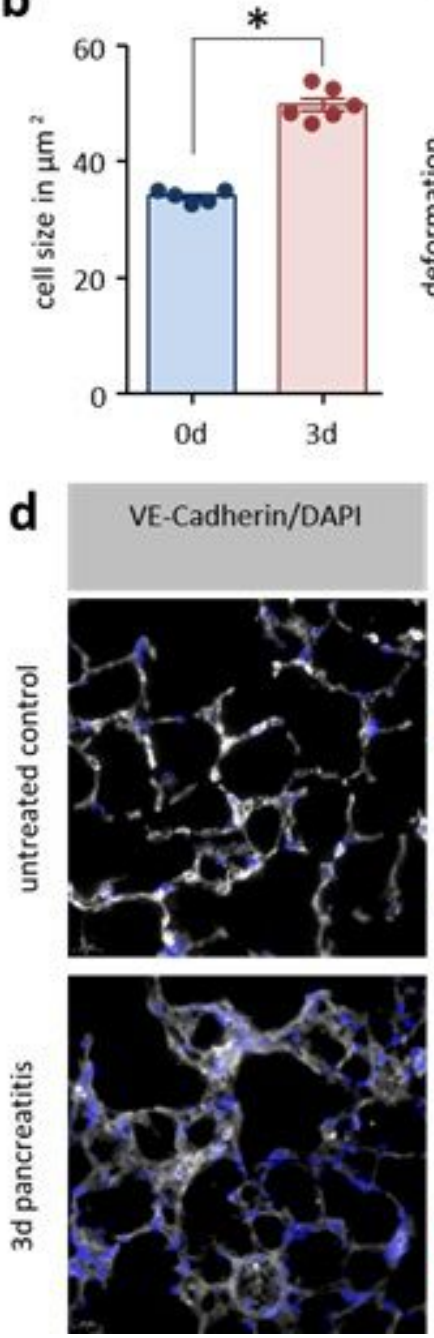

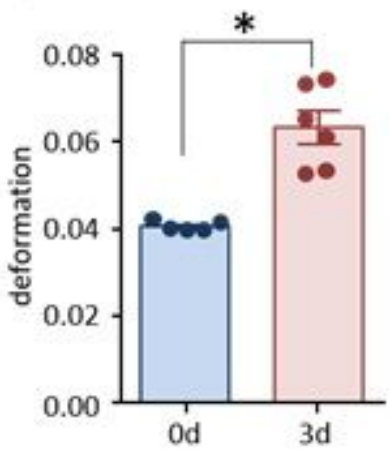

$3 d$ acute pancreatitis

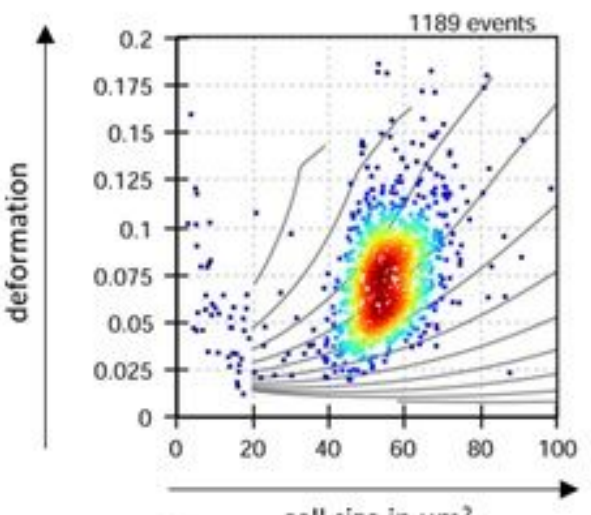

C

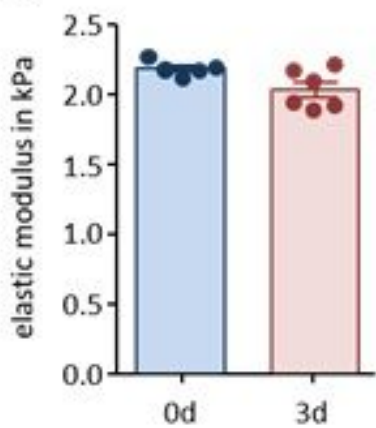

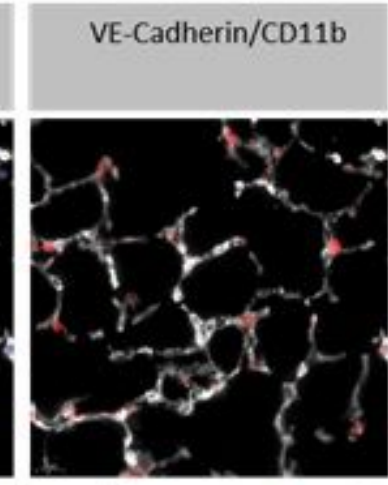
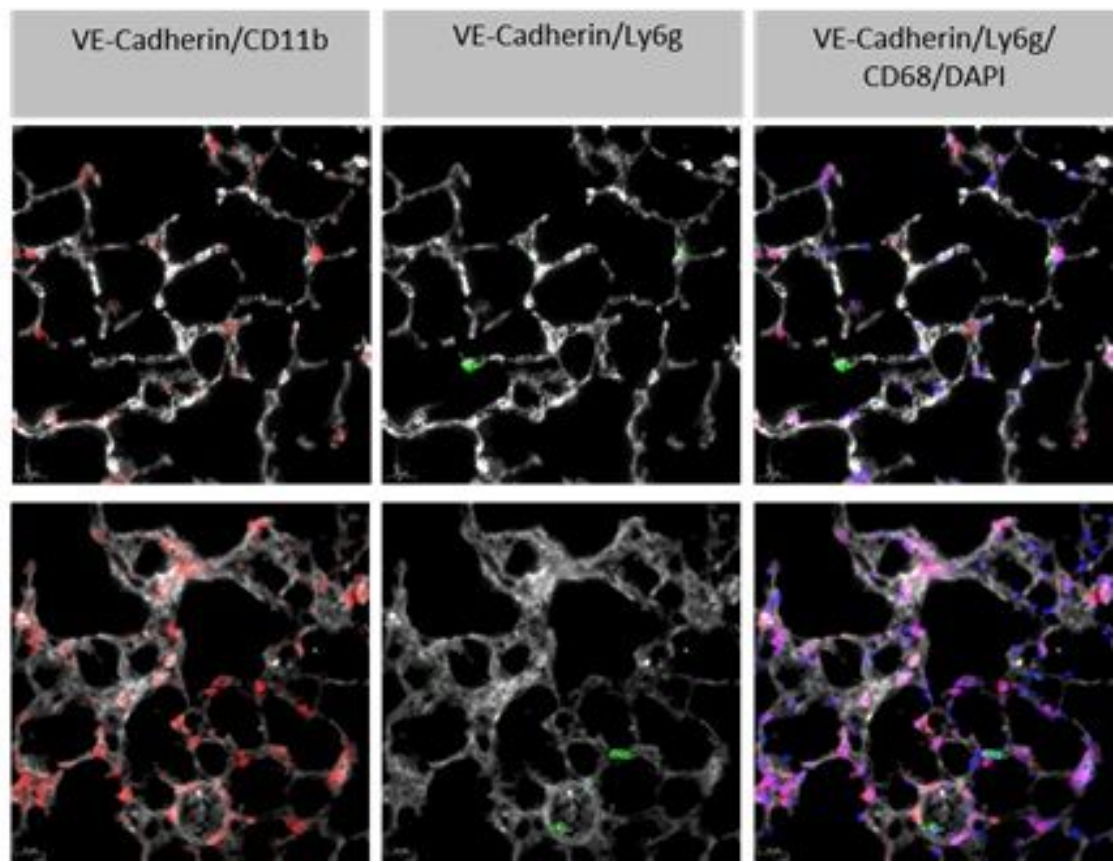

Figure 7

Monocytes isolated from lung tissue of pancreatitis animals are characterized by increased cell size without changes in their deformation. Monocytes (reflects CD11b+/Ly6g-cells) from lung tissue of mice with and without SAP were isolated and analysed by RT-FDC. Deformation vs. cell size scatter plots of monocytes from lung showed the same mechanical characteristics as blood CD 11b+/Ly6g-cells (Figure 6) (a). Statistical analysis of experimental replicates indicates a significant increase in cell size and 
deformation (b). No significant change in elastic modulus is observed (c). All graphs represent 5 or more animals per group, Statistical significant difference was tested by unpaired, two tailed students t-test. Fluorescent labelling of lung tissue confirmed localisation of CD11b+ cells in the capillary system of the lung. After the induction of pancreatitis $\mathrm{CD} 11 \mathrm{~b}+$ monocytes (red) represent the majority of detectable cells in lung tissue, whereas neutrophils (green) were observed in much lower numbers (d). Monocytes are located within VE-Cadherin (white) positive areas, which indicates a vascular redistribution. RT-FDC data has been analysed using linear mixed models $(*, p<0.05)$.

\section{Supplementary Files}

This is a list of supplementary files associated with this preprint. Click to download.

- SupplementaryFigures.pdf

- NCOMMS2113677rs.pdf 